\title{
Magnetic-topology evolution in NOAA AR 10501 on 2003 November 18
}

\author{
A. V. Oreshina, I. V. Oreshina, and B. V. Somov
}

\begin{abstract}
Sternberg Astronomical Institute, Moscow State University, Universitetski prospekt, 13, 119991 Moscow, Russia
\end{abstract} e-mail: ivo@sai.msu.ru

Received 1 July 2011 / Accepted 17 December 2011

\begin{abstract}
Context. NOAA AR 10501 produced three flares on 2003 November 18. Two of them were associated with coronal mass ejections (CMEs).

Aims. We model the magnetic-field structure of the active region, study the magnetic-topology evolution, and propose a scenario of the observed events.

Methods. The coronal magnetic field is reconstructed using a topological model (also called magnetic-charge model). We present an automatic method of choosing the magnetic charges for the case where the charges are located beneath the photosphere. The new method improves quantitative analysis of magnetograms and makes processing faster.

Results. We demonstrate that coronal conditions became more favourable for magnetic reconnection before the flaring events. It is also shown that the magnetic-field configuration at the time of both CMEs was critical, close to what is called "topological trigger". We assume that the topological trigger played a key role in the initiation of these CMEs.
\end{abstract}

Key words. Sun: magnetic topology - Sun: flares - Sun: coronal mass ejections (CMEs)

\section{Introduction}

According to current understanding, a solar flare results from the reconnection of magnetic fields, i.e. the magnetic reconnection. There remains, however, considerable debate on which factors make some magnetic configurations more likely to flare than others. The exact relationship between flares and CMEs is also the subject of many works.

A powerful tool for answering these questions is a topological model, also called the magnetic-charge model. A review of this topic can be found in Somov (2006). It assumes that a real magnetic field is modelled by the one created by "effective charges", or "sources". This approach allows us to reconstruct the coronal structure and to distinguish different magnetic fluxes and the places where these fluxes interact among themselves, i.e. to study magnetic-field topology. The boundary surfaces of the fluxes are called the separatrix surfaces or "separatrices". They intersect among themselves along field lines called the limiting field lines or "separators" (Sweet 1969; Syrovatskii 1981).

The charges can be located either beneath the photospheric plane or on this plane. The advantage of the models from the first category is a relatively realistic approximation of an observed photospheric field. These models are also good at representing the shapes of flare ribbons observed in $\mathrm{H} \alpha$, hard X-ray, and EUV ranges (Gorbachev \& Somov 1989; Mandrini et al. 1991; Démoulin et al. 1993; Somov et al. 2005). They have allowed us to reveal some causes of the observed magnetic-field imbalance in solar active regions (Oreshina \& Somov 2006) and to interpret drastic changes in the magnetic fields associated with solar flares (Oreshina \& Somov 2008).

The models from the second category assume that singular magnetic sources are located on the photospheric plane (Barnes et al. 2005; Des Jardins et al. 2009; Kazachenko et al. 2010; Longcope et al. 2010). These models are well suited to quantitative analysis because exactly half of the flux from a charge is located above the photosphere. On the other hand, the vertical component of a model magnetogram is nought everywhere except points where charges are located, so a visual comparison between an observed magnetogram and a model one is impossible for this category.

Démoulin et al. (1994) have developed an automatic algorithm for positioning sources beneath the photosphere at different depths. In comparison with models where all the sources are located on the same plane, the differences in the topology are not crucial; however, such an algorithm takes more computational time and makes the following analysis of the obtained topology more complicated. An automatic algorithm for partitioning magnetograms into charge regions was proposed by Barnes et al. (2005) for the models from the second category.

The main aim of our paper is to propose an automatic algorithm for choosing the magnetic charges located beneath the photosphere on one horizontal plane. It will allow us to make the model the clearest and the simplest and to join the advantages of both categories: $(i)$ the model photospheric magnetic field will approximate the observed one closely, and (ii) a quantitative and fast analysis of a large number of magnetograms will be possible, too.

Then we apply the method for modelling a real active region in order to propose a scenario of observed eruptive events. We focus attention on the trigger of flares and CMEs by taking topological characteristics of the magnetic-field structure into account.

Knowing the location of reconnection is a key to understanding a flare trigger. According to current understanding, the magnetic reconnection mechanism at a separator transforms magnetic energy into thermal and kinetic energy of plasma and accelerated particles. Accelerated electrons follow field lines crossing a region of primary energy release and descend to the 


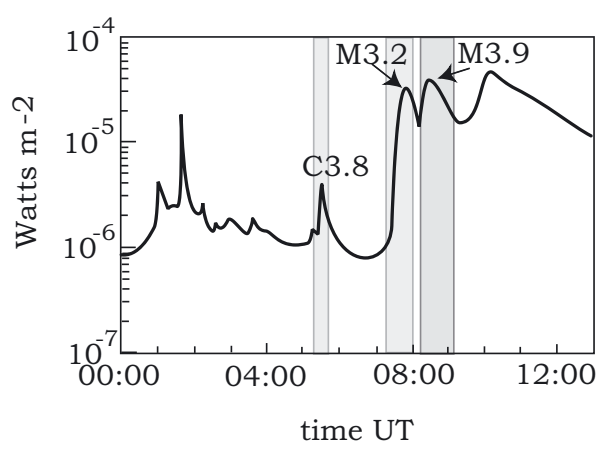

Fig. 1. Temporal evolution of the solar X-ray flux observed by GOES 12 in 1.0-8.0 $\AA$ bandwidth on 2003 November 18. The flares occurred in AR 10501 are marked in grey.

chromosphere where they produce hard X-ray (HXR) radiation. Des Jardins et al. (2009) notice a remarkable visual relationship between the spine lines and HXR footpoint tracks. They also demonstrate that there are spine lines that are not associated with HXR footpoints (Fig. 5 in their paper). Restante et al. (2009) have studied the relations between quasi-separatrix layers (QSLs), which are regions where there is a drastic change in field line linkage and in skeletons, which are composed of null points, spine field lines, and fans. They have shown that spines, as well as specific portions of fans are good predictors for the location of QSL footprints, and therefore of flare ribbons. They also pay attention to the fact that only some portions of the skeleton are found to be related to flare ribbons. In our paper we propose an explanation of these results using active region NOAA 10501, as an example. Moreover, we focus our attention on the trigger of flares and CMEs taking topological characteristics of magneticfield structure and evolution into account.

The paper is organised as follows. In Sect. 2, we describe the observational data of AR NOAA 10501. Then, in Sect. 3, a new automatic method is presented for choosing magnetic charges; it is applied to constructing the topological model of the AR under consideration. In Sect. 4, the changes in the magnetic field are analysed from 00:00 UT to 11:12 UT. The results are discussed in Sect. 5. In the last section, we formulate our conclusions.

\section{Observations of NOAA AR 10501 on 2003 November 18}

On 2003 November 18, AR 10501 was located near the centre of the solar disc (N 03, E 08). Figure 1 shows the temporal evolution of the solar X-ray flux observed by Geostationary Operational Environmental Satellite (GOES). Five flares occurred on that day. Three of them were observed to originate in the AR under consideration: $\mathrm{C} 3.8$ at 05:25 UT, M3.2 at 07:52 UT, and M3.9 at 08:30 UT. The second and the third flares were associated with two CMEs, detected by Large Angle and Spectrometric Coronagraph (LASCO) on board SOHO. The first CME was detected in the $\mathrm{C} 2$ field-of-view at 08:06 UT and the second one was observed at 08:50 UT. Two days later, these CMEs produced the most powerful geomagnetic storm in solar cycle \# 23 (Ermolaev et al. 2005; Srivastava et al. 2009; Chandra et al. 2010).

Figure 2 presents the line-of-sight magnetogram obtained by the Michelson Doppler Imager (MDI) on-board SOHO at 00:00 UT. We show the five areas where magnetic field was the most intense: two areas of southern (negative) polarity and three areas of northern (positive) polarity. The maximal absolute value

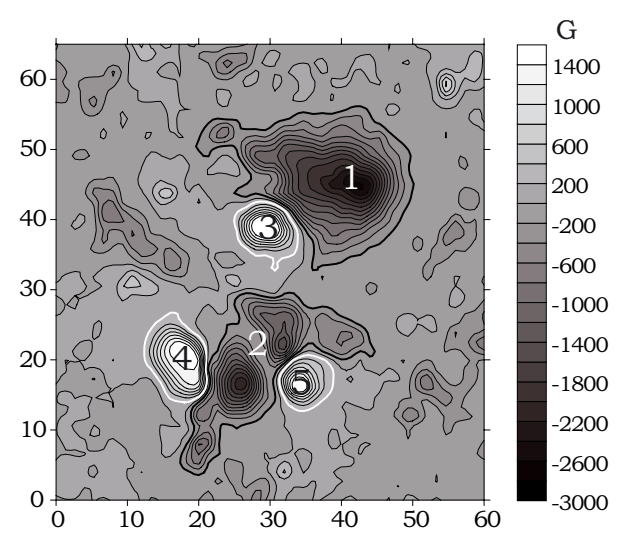

Fig. 2. Magnetogram obtained by MDI/SOHO at 00:00 UT on 2003 Nov. 18. The white (black) thick lines are $200 \mathrm{G}(-200 \mathrm{G})$ levels. A length unit corresponds to one MDI pixel $\left(1.45 \times 10^{8} \mathrm{~cm}\right)$.

of the field for both polarities was about $3000 \mathrm{G}$. We have chosen $200 \mathrm{G}$ as a boundary intensity level for these areas. Since our aim is to study the magnetic-flux evolution, this threshold allows us not to analyse the small-scale changes due to variations in atmospheric seeing and uncorrelated noise. We also do not consider small areas covering just a few pixels, because small fluxes seem to us unlikely to contribute much to the global topology, so we study the evolution of these five areas, which include a significant part of the global (whole AR) magnetic flux and which determine its large-scale topology.

\section{Method for choosing charges for topological model}

The 3D magnetic field created by charges is described by the formula

$\boldsymbol{B}(x, y, z)=\sum_{i=1}^{N} \frac{e_{i}}{\left|\boldsymbol{r}-\boldsymbol{r}_{i}\right|^{2}} \cdot \frac{\boldsymbol{r}-\boldsymbol{r}_{i}}{\left|\boldsymbol{r}-\boldsymbol{r}_{i}\right|}$,

where $N$ is the number of charges, $e_{i}$ their intensities, and $\boldsymbol{r}_{i}=$ $\left(x_{i}, y_{i}, z_{i}\right)$ their radius vectors. The $x$-axis is directed to the west, the $y$-axis to the north, and the $z$-axis is directed upwards from the solar surface. The $z=0$ plane corresponds to the photosphere. We assume that all the charges are located beneath the photosphere at the same depth: $z_{i}=$ const. $=z_{0}$ for every $i$. As proposed in the previous section, $N=5$ for this AR, so there are 16 unknowns: $\left(x_{i}, y_{i}\right), e_{i}$ where $i=1 \ldots 5$, and $z_{0}$.

First, we calculate charge positions $\left(x_{i}, y_{i}\right)$ as the "centre of mass" of every area $R_{i}$ :

$x_{i}=\frac{\sum_{k \in R_{i}} x_{k} \psi_{k}}{\sum_{k \in R_{i}} \psi_{k}}, \quad y_{i}=\frac{\sum_{k \in R_{i}} y_{k} \psi_{k}}{\sum_{k \in R_{i}} \psi_{k}}$.

Here $\psi_{k}$ is the observed magnetic flux through the area on the photosphere that corresponds to MDI pixel $k$, and $x_{k}$ and $y_{k}$ are the coordinates of this pixel. The summation is over all pixels of area $R_{i}$. 
Table 1. Intensities and coordinates of the magnetic-field charges for modelling the magnetogram obtained at 00:00 UT.

\begin{tabular}{ccccc}
\hline \hline$i$ & $e_{i}$ & $x_{i}$ & $y_{i}$ & $z_{0}$ \\
\hline 1. & -98317 & 39.5 & 46,9 & -4 \\
2. & -79537 & 29.2 & 19.5 & -4 \\
3. & 30834 & 30.4 & 39.6 & -4 \\
4. & 36272 & 18.3 & 21.0 & -4 \\
5. & 35496 & 35.7 & 17.8 & -4 \\
\hline
\end{tabular}

Then, we find the charge intensities $e_{i}$ from the equality of the observed and the model magnetic fluxes for each area $R_{i}$ :

$$
\left\{\begin{array}{l}
\sum_{k \in R_{1}}\left[\sum_{i=1}^{N} \frac{e_{i}}{\left|\boldsymbol{r}_{k}-\boldsymbol{r}_{i}\right|^{2}} \frac{\left(z_{k}-z_{0}\right)}{\left|\boldsymbol{r}_{k}-\boldsymbol{r}_{i}\right|}\right] \cdot s=\Psi_{1} \\
\sum_{k \in R_{5}}\left[\sum_{i=1}^{N} \frac{e_{i}}{\left|\boldsymbol{r}_{k}-\boldsymbol{r}_{i}\right|^{2}} \frac{\left(z_{k}-z_{0}\right)}{\left|\boldsymbol{r}_{k}-\boldsymbol{r}_{i}\right|}\right] \cdot s=\Psi_{5} .
\end{array}\right.
$$

Here $\Psi_{i}$ is the observed magnetic flux in the area $R_{i}, s$ the surface of an MDI pixel: $s=2.1 \times 10^{16} \mathrm{~cm}^{2}$. There are five equations with six unknowns in this system: $e_{1} \ldots e_{5}$ and $z_{0}$. We then consider depth $z_{0}$ as a free parameter. Different values of $z_{0}$ will correspond to different sets of intensities $e_{1} \ldots e_{5}$.

Let us choose the set that approximates the global magnetic flux in the best way. In other words, we want to minimise the difference between model and observed fluxes on the AR scale:

$$
\left\{\begin{array}{l}
\sum_{k_{+}}\left[\sum_{i=1}^{N} \frac{e_{i}}{\left|\boldsymbol{r}_{k}-\boldsymbol{r}_{i}\right|^{2}} \frac{\left(z_{k}-z_{0}\right)}{\left|\boldsymbol{r}_{k}-\boldsymbol{r}_{i}\right|}\right] \cdot s-\Psi_{+}=\Delta_{+} \\
\sum_{k_{-}}\left[\sum_{i=1}^{N} \frac{e_{i}}{\left|\boldsymbol{r}_{k}-\boldsymbol{r}_{i}\right|^{2}} \frac{\left(z_{k}-z_{0}\right)}{\left|\boldsymbol{r}_{k}-\boldsymbol{r}_{i}\right|}\right] \cdot s-\Psi_{-}=\Delta_{-} .
\end{array}\right.
$$

The summation in the first formula is over all magnetogram pixels with positive magnetic field, the one in the second formula is over all pixels with negative magnetic field, and $\Psi_{+}\left(\Psi_{-}\right)$is the observed positive (negative) magnetic fluxes in the whole AR.

Our experience shows that a model magnetogram approximates an observed one well if the charges are located at the depth of several units, not more than ten. This part of the method does not take much time.

The charge coordinates and intensities for the magnetogram from Fig. 2 are presented in Table 1. The corresponding model magnetogram is shown in Fig. 3. The output of this model provides the global magnetic flux in agreement with the observational values at about $90 \%$ (92\% for positive-flux approximation and $88 \%$ for negative-flux approximation). We process other magnetograms of the AR keeping the same depth $z_{0}$. All we need to recalculate are the coordinates of charges $\left(x_{i}, y_{i}\right)$ (from formula 2) and their intensities (from system 3 ).

\section{Magnetic-field topology}

\subsection{Separators and separatrix surfaces}

Determination of the magnetic charges allows the topology to be analysed. Figure 4 shows the vector field in the charge plane $z=z_{0}=-4$. Solid lines are the intersections of the separatrix surfaces with this plane. Des Jardins et al. (2009) and Restante et al. (2009) conclude that flaring ribbons correspond to some

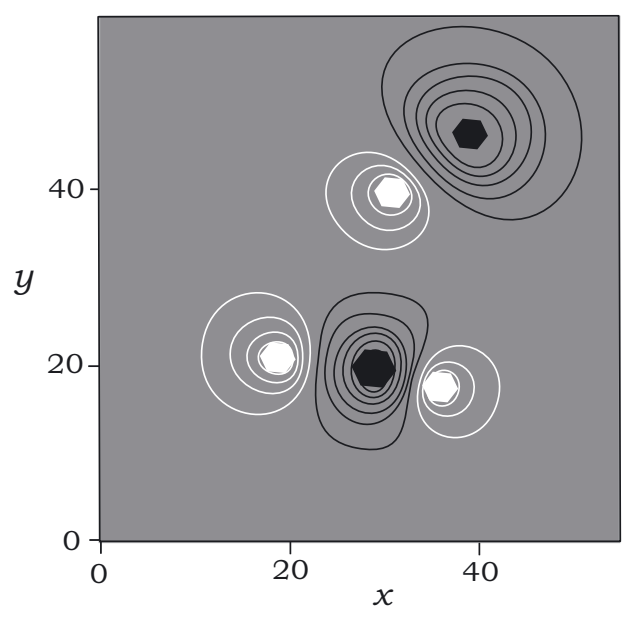

Fig. 3. Model magnetogram corresponding to 00:00 UT.

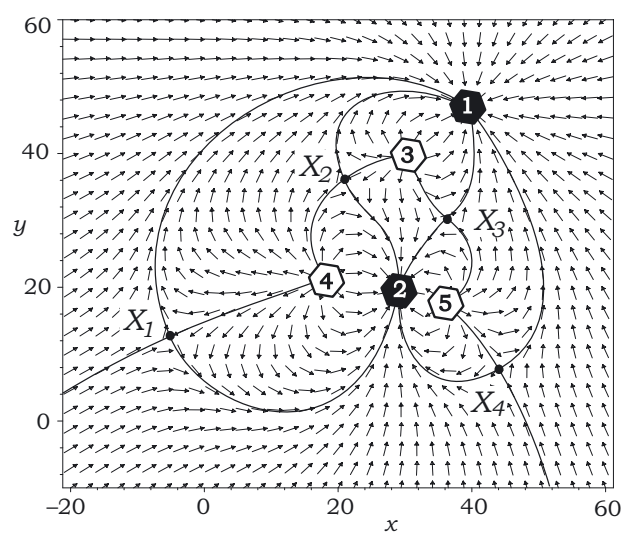

Fig. 4. Vector field created by charges in the charge plane. Solid lines are the intersections of the separatrix surfaces with this plane, and $X_{1}$, $X_{2}, X_{3}$, and $X_{4}$ are the null points.

parts of these lines. There remains the question of which parts of such lines correspond to the location where flare ribbons might be found. The answer consists in the necessity of searching separators. It is here, in the vicinity of the separators, that the magnetic-field structure makes the reconnection possible. Particles accelerated by reconnection come down to the photosphere where they produce hard X-ray bremsstrahlung radiation.

As is known, separator endpoints are null points in the magnetic field, i.e. the points where the magnetic field vanishes. However not every null point is a separator endpoint. As one can see in Fig. 4, there are four points where the field is nought in the charge plane: $X_{1}, X_{2}, X_{3}$, and $X_{4}$. Figure 5 demonstrates that there is a separator connecting the points $X_{2}$ and $X_{3}$. Indeed, there is a line connecting these two points (Fig. 5, left), and the vector field in the vertical plane perpendicular to the straight segment $\left(X_{2}, X_{3}\right)$ contains an $X$-type null point (Fig. 5, right). This is one of structures that enable magnetic reconnection (Oreshina \& Somov 1998; Hornig \& Priest 2003; De Moortel \& Galsgaard 2006; Parnell et al. 2010).

Analogically, it is possible to show the existence of the separator $X_{3} X_{4}$, so point $X_{3}$ is a common endpoint of both separators. Point $X_{1}$ does not correspond to any separator. Finally, there are two separators in this AR. The observed flares can be explained by the magnetic reconnection processes on them.

Searching separators is still a difficult problem that needs the investigation of a 3D magnetic structure. To develop an 


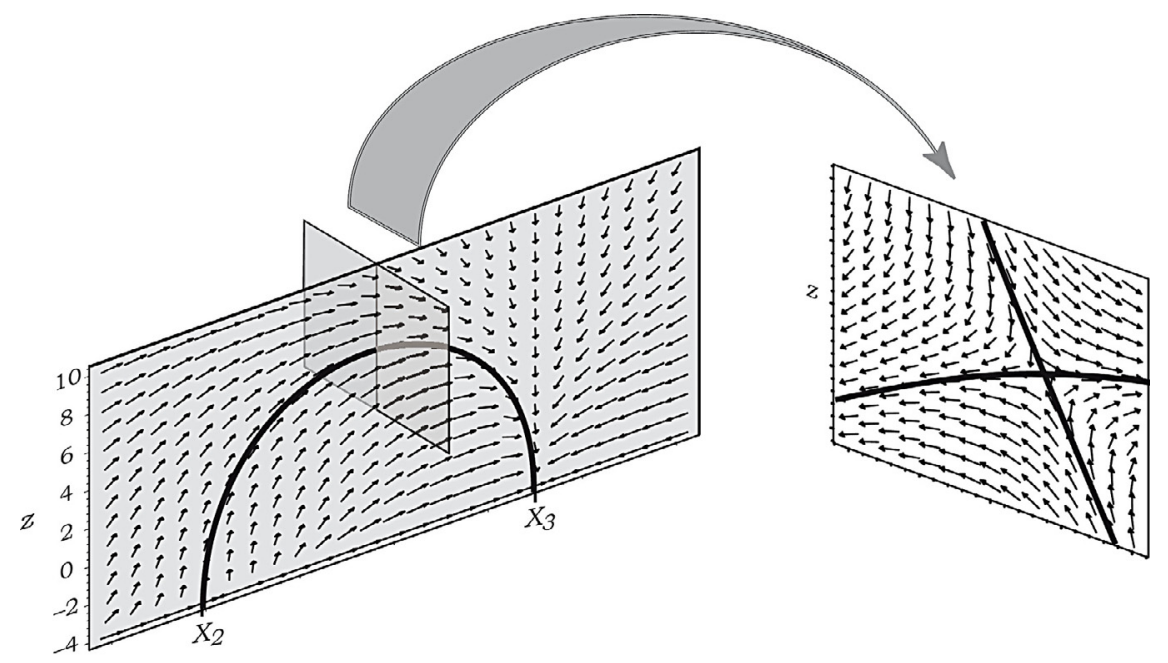

Fig. 5. Left: vector field in the vertical plane containing points $X_{2}$ and $X_{3}$; there is a field line connecting these points. Right: vector field in the vertical plane perpendicular to the straight segment $\left(X_{2}, X_{3}\right)$; there is an $X$-type null point - a structure making reconnection possible.

automatic and effective method for this part of the topological analysis is one of the aims of our future studies.

\subsection{Topology evolution}

The aim of this section is to investigate the conditions on separators in order to answer the question of why one magnetic configuration is more likely to reproduce a flare than another one. Somov \& Titov (1985a,b) and Somov (2006, Sect. 6.2.2) demonstrated that a strong longitudinal (along the separator) component of the magnetic field can considerable limit energy release in a reconnecting current layer (RCL). Indeed, decreasing the longitudinal magnetic field increases the compressibility of plasma flowing into the RCL. As a result, the width of the layer decreases, which leads to increasing the reconnection rate. The procedure aiming to find where the flare ribbons might be located should therefore contain two steps: (1) searching separators and their footprints in the photospheric plane, (2) analysing physical conditions on separators (estimation of magnetic-strength value).

Oreshina \& Somov (2009) proposed a topological-model parameter that allows the longitudinal magnetic field on a separator to be judged. The lower its value, the better the conditions for magnetic reconnection. As an example, they constructed the topological models of AR NOAA 9077 for 2000 July 12 and 14, i.e. two days before the Bastille Day flare and the flare day. They showed that there were two separators and that the coronal conditions for reconnection on both of them improved significantly before the flare. In the present paper, we investigate the evolution of this parameter on the scale of several hours before and after the flares and CMEs.

The parameter under consideration is $\lambda_{z}$. It is calculated at the null points, which are the separator endpoints as follows. One of the characteristics of an arbitrary null point $X_{0}$ is the matrix with elements (e.g. Somov 2008a)

$M_{\alpha \beta}\left(X_{0}\right)=-\frac{\partial^{2} \varphi\left(X_{0}\right)}{\partial \alpha \partial \beta}$.

Here $\alpha$ and $\beta$ are indexes that can take values $x, y$, and $z$, and $\varphi$ is the potential of the field (1):

$\varphi(x, y, z)=\sum_{i=1}^{N} \frac{e_{i}}{\left|\boldsymbol{r}-\boldsymbol{r}_{0}\right|}$.

A138, page 4 of 6
As a result, (4) is the matrix of magnetic-field gradients at the null point $X_{0}$. We are interested in the eigenvalue $\lambda_{z}$ corresponding to the vertical eigenvector of (4), i.e. in the gradient value along a separator. If the separator length does not significantly change with time, the lower the gradient absolute value at separator endpoints, the lower the absolute value of the magnetic field at the separator, the better the conditions for reconnection.

We let $\lambda_{z}\left(X_{1}\right), \lambda_{z}\left(X_{2}\right), \lambda_{z}\left(X_{3}\right)$, and $\lambda_{z}\left(X_{4}\right)$ be the parameters characterising the points $X_{1}, X_{2}, X_{3}$, and $X_{4}$, respectively. We study a set of eight magnetograms of the AR NOAA $10501 \mathrm{ob}-$ tained by MDI/SOHO on 2003 November 18 between 00:00 UT and 11:12 UT with 96 min intervals. The changes at the null points are presented in Fig. 6, with the moments of the three flares under consideration. These are the same flares as in Fig. 1. Parameter $\lambda_{z}\left(X_{3}\right)$ stayed negative during the reporting period; the figure shows its absolute values.

Our calculations show that the parameter $\lambda_{z}\left(X_{1}\right)$ remained almost constant. There were oscillations of the parameter $\lambda_{z}\left(X_{2}\right)$ with an amplitude of less than two units. Analogically, the parameter $\lambda_{z}\left(X_{4}\right)$ varied within the limits of 2.5 units. The most significant changes were observed at point $X_{3}$. Here the parameter $\left|\lambda_{z}\left(X_{3}\right)\right|$ decreased by 6.4 units (from 13.0 to 6.6). Note that $X_{3}$ is a common endpoint of both separators. The first flare (05:25 UT) occurred on the decreasing phase of this parameter. The second and the third flares (07:52 UT and 08:30 UT) coincide with its deep minimum.

Figure 7 presents the maximum values of magnetic field at both separators for each of the eight time points. We have connected the points by two lines to separate the processes on two separators better. One can see that two flares occurred in the vicinity of the deepest magnetic field minimum on the separator $X_{3} X_{4}$. These two events also coincide with a minimum on the separator $X_{2} X_{3}$ even if not as deep. It allows the stronger energy to be released during flares. Indeed, the first flare was that of $\mathrm{C}$ class, while the second and the third ones were $\mathrm{M}$ class.

Comparing Figs. 6 and 7 shows some similarity between the evolution of the parameters $\lambda_{z}$ and that of the magnetic field values on separators. This confirms our suggestion that the lower the parameter $\lambda_{z}$, the better the conditions for the reconnection. This similarity is not a perfect correlation because the parameters $\lambda_{z}$ at two endpoints of a separator change differently. But we propose to pay special attention to the separators for which the parameters $\lambda_{z}$ decreases significantly. 
A. V. Oreshina et al.: Magnetic topology of NOAA AR 10501
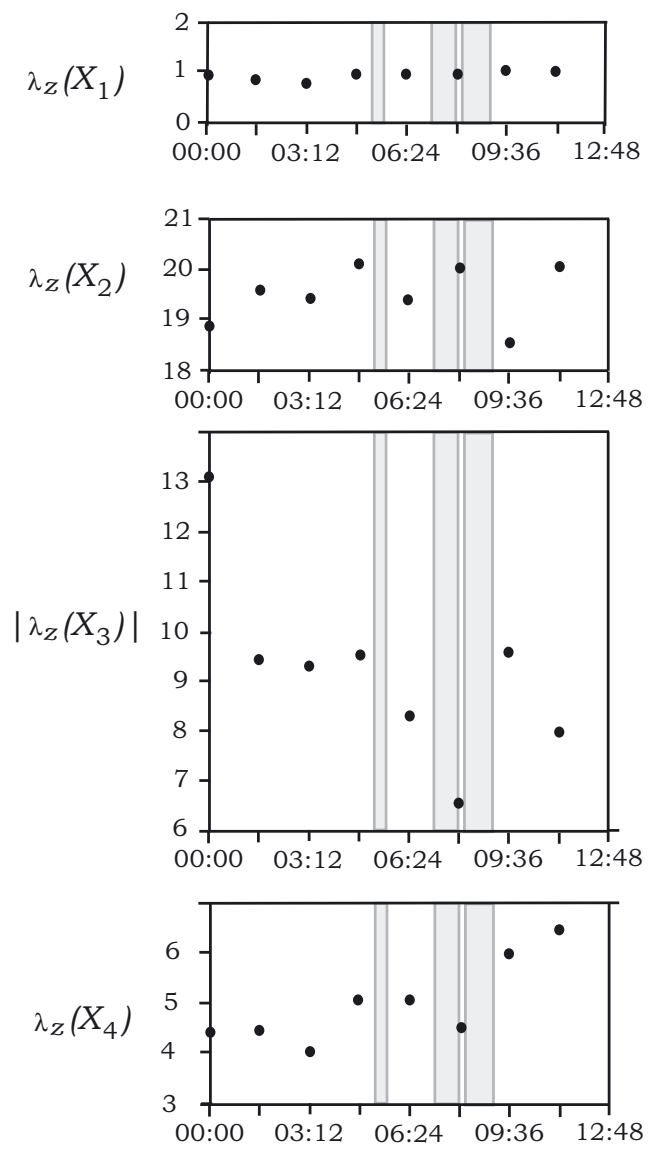

Fig. 6. Changing values of the parameters $\lambda_{z}\left(X_{1}\right), \lambda_{z}\left(X_{2}\right),\left|\lambda_{z}\left(X_{3}\right)\right|$, and $\lambda_{z}\left(X_{4}\right)$ with time. The moments of the three flares under consideration are marked in grey. These are the same flares as in Fig. 1.

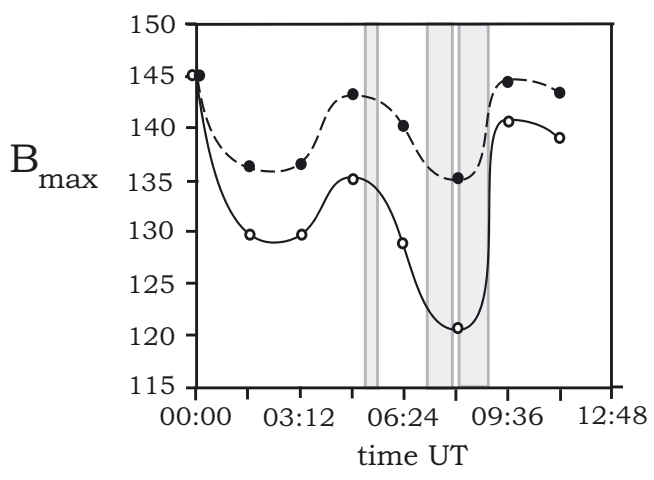

Fig. 7. Maximum values of magnetic field (in Gauss) on both separators for each of the eight time points. Black and white circles correspond to the separators $X_{2} X_{3}$ and $X_{3} X_{4}$, respectively.

This result can be used to develop a reliable and physically justified method for predicting solar flares. A more detailed study of the relationship between parameters $\lambda_{z}$, the magnetic field on separators, and flare trigger will be the focus of our future work. Parameter $\lambda_{z}$ is calculated from a 2D magnetic field in the plane of charges. In comparison with those in a 3D magnetic structure, such calculations are thus simpler and faster. One can first calculate values $\lambda_{z}$ at all null points and then search for the separators connecting those that show the evolution towards the reconnection. It will be faster than searching all the separators of an active region.
Table 2. Intensities and coordinates of the magnetic-field charges for modelling the magnetogram obtained at 08:00 UT.

\begin{tabular}{ccccc}
\hline \hline$i$ & $e_{i}$ & $x_{i}$ & $y_{i}$ & $z_{0}$ \\
\hline 1. & -101031 & 39.5 & 46.9 & -4 \\
2. & -84850 & 27.7 & 18.6 & -4 \\
3. & 32098 & 29.3 & 39.2 & -4 \\
4. & 40712 & 17.2 & 20.7 & -4 \\
5. & 32987 & 35.5 & 17.9 & -4 \\
\hline
\end{tabular}

\subsection{Topological trigger}

Syrovatskii \& Somov (1980) showed that slow motions of the photospheric field can lead to a rapid change in the coronal magnetic structure. The term "topological trigger" was introduced by Gorbachev et al. (1988). They used the simplest 3D model of potential magnetic field and pointed out the appearance of a new null point in the corona and its fast motion along the separator above the photosphere. Inverarity \& Priest (1999) demonstrate the theoretical existence of stable coronal nulls, but do not discuss in detail the mechanisms which cause them to be born through a bifurcation. Brown \& Priest (2001) explored the topology of the simplest configuration due to four sources with a coronal null and a bifurcation that causes such a null point to be born or to die.

Meanwhile, the question of precisely how the topological trigger materialises under actual conditions in solar active regions remained unclear. Somov (2008a,b) showed that the topological trigger effect should be taken into account when large eruptive flares are modelled. This possibility was illustrated by the example of an extended 1B/M4 flare on 1980 November 5 in AR 2776. The 3D topological trigger effect is not a resistive instability that leads to changing the topology of the field configuration from pre- to post-reconnection state. On the contrary, the topological trigger is a quick change in the global topology that dictates the fast reconnection in large eruptive flares (Somov 2008b; Oreshina \& Somov 2009).

We now come back to the solar flares under consideration and investigate in detail the magnetic-field structure on the separators at 08:00 UT, i.e. between the two last flares. The locations of the charges and their intensities corresponding to this moment are presented in Table 2. The comparison with the initial moment (00:00 UT, Table 1) shows that the charges moved within two pixels. The intensities of the first four charges increased, while that of the fifth charge decreased. In this section, we demonstrate that the magnetic-field configuration became critical so that even minor changes in the charge characteristics could lead to drastic changes in the coronal magnetic field.

Figure 8 (top) shows the vector field in the plane of the separators $X_{2} X_{3}$ and $X_{3} X_{4}$ at 08:00 UT. If we only move the charge 3 along the $x$-axis from the point $x_{3}=29.3$ to the point $x_{3}=29.4$, keeping the coordinates and intensities of other charges, the sign of the parameter $\lambda_{z}\left(X_{3}\right)$ will be changed. It means changing the magnetic-field direction along both separators at the endpoint $X_{3}$ and, therefore, changing the magnetic structure in the vicinity of both separators. These changes are presented in Fig. 8 (middle). A new null point is born from the point $X_{3}$ on the separator $X_{2} X_{3}$ at the height of $z=0.06$, i.e. at the photospheric level.

Possible changes in charge characteristics, even the most minor ones, lead to the fast motion of this null point along the separator. As an example, Fig. 8 (bottom) presents the magneticfield configuration in the vicinity of separators for the case when charge 3 is moved to the point $\left(x_{3}, y_{3}\right)=(30.0,37.0)$. The new 

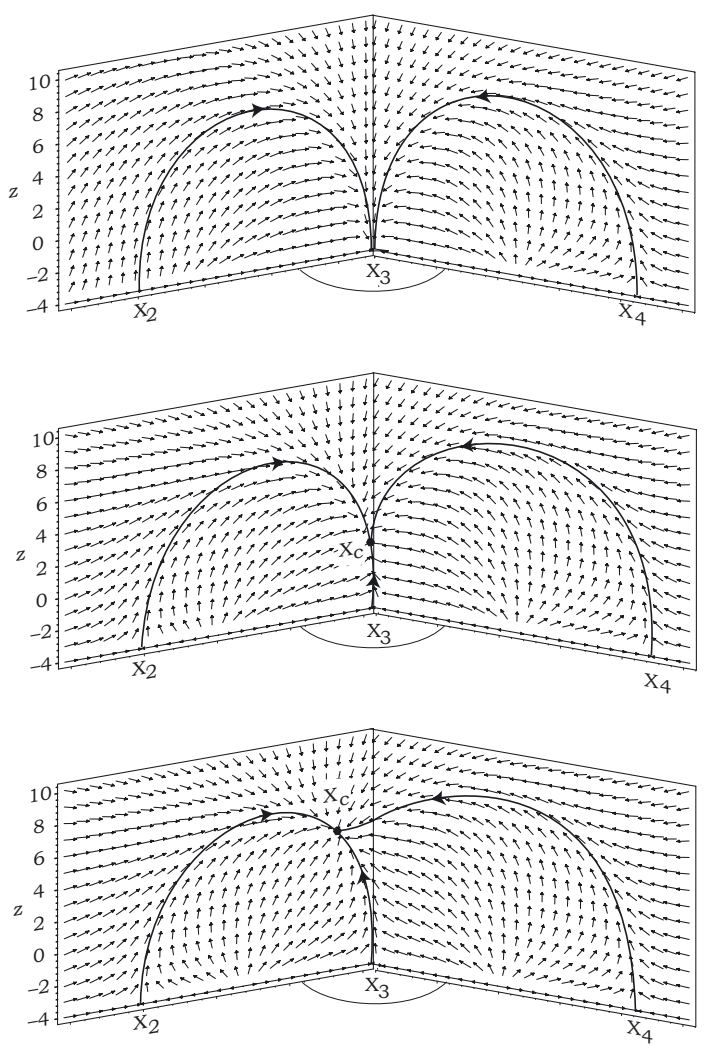

Fig. 8. Vector field in the plane of the separators $X_{2} X_{3}$ and $X_{3} X_{4}$. Top: configuration corresponding to the system parameters presented in Table 2. Middle: the same for the case when charge 3 is moved to the point $x_{3}=29.4 . X_{\mathrm{c}}$ is a new null point. Bottom: the same for the case when charge 3 is moved to the point $\left(x_{3}, y_{3}\right)=(30.0,37.0)$.

null point moved up to the height of $z=4.5$. Here, in the corona, a null point on a separator implies the most favourable conditions for magnetic-field reconnection and makes this process the most powerful (Somov 2006). However, this is not the whole story. As seen from Fig. 8, the presence of a coronal null point makes the magnetic-field structure instable. Even small variations in the magnetic field in the photosphere involve drastic changes in the coronal magnetic field on both separators, which can cause CMEs.

Also the existence of a coronal null point plays a key role in a number of models for eruptive events, for example, breakout model for CMEs (Antiochos et al. 1999). Our method for analysing the topology of the large-scale magnetic field of an AR allows us to find coronal null points situated on separators, i.e., precisely where the magnetic energy is accumulated before a flare, and is converted into the particle energy during a flare.

We do not claim that the movement of exactly charge 3 caused the topological trigger, hence CMEs in this AR. We just demonstrate that some critical state could be reached easily and trigger drastic changes in the coronal magnetic-field structure in the vicinity of two separators.

\section{Conclusions}

A method was proposed for automatically choosing the magnetic charges for topological modelling solar ARs. It improves quantitative analysis of magnetograms and makes processing faster, which in turn allows the magnetic-field evolution to be analysed on small timescales.

The method is applied to NOAA AR 10501, which produced three flares and two CMEs on 2003 November 18. Our model suggests the following possible scenario for these events. The magnetic field structure was characterised by the presence of two separators. The conditions on them became more favourable for magnetic reconnection between 00:00 UT and 08:00 UT, which caused three flares. The longitudinal magnetic field decreased significantly along both separators at about 08:00 UT when two flares occurred. As a consequence, the second and the third flares were more powerful than the first one (M-class in contrast with C-class). At 08:00 UT, the magnetic-field structure was very close to a critical state. Even minor changes in photospheric magnetic field could lead to rapid changes in the corona in the vicinity of both separators, i.e. to a topological trigger that could cause two CMEs.

Acknowledgements. We are grateful to the anonymous referee, whose constructive comments have improved this paper. We thank the SOHO team for the availability of the satellite data. We took the magnetograms from the web site http://soi.stanford.edu/data/. This work was supported by the RFBR grant No. 11-02-00843-a.

\section{References}

Antiochos, S. K., DeVore, C. R., \& Klimchuk, J. A. 1999, ApJ, 510, 485 Barnes, G., Longcope, D. W., \& Leka, K. D. 2005, ApJ, 629, 561

Brown, D. S., \& Priest, E. R. 2001, A\&A, 367, 339

Chandra, R., Pariat, E., Schmieder, B., et al. 2010, Sol. Phys., 261, 127

Démoulin, P., van Driel-Gesztelyi, L., Schmieder, B., et al. 1993, A\&A, 271, 292

Démoulin, P., Mandrini, C. H., Rovira, M. G., et al. 1994, Sol. Phys., 150, 221

Des Jardins, A., Canfield, R., Longcope, D., et al. 2009, ApJ, 693, 1628

De Moortel, A., \& Galsgaard, K. 2006, A\&A, 451, 1101

Ermolaev, Yu. I., Zelenyi, L. V., Zastenker, G. N., et al. 2005, Geomagnetism and Aeronomy, 45, 20

Gorbachev, V. S., \& Somov, B. V. 1989, Sov. Astron. AJ, 33, 57

Gorbachev, B. S., KelŠner, S. R., Somov, B. V., \& Schwarz, A. S. 1988, Sov. Astron. AJ, 32, 308

Hornig, G., \& Priest, E. R. 2003, Phys. Plasmas, 10, 2712

Inverarity, G. W., \& Priest, E. R. 1999, Sol. Phys., 186, 99

Kazachenko, M. D., Canfield, R. C., Longcope, D. W., \& Qiu, J. 2010, ApJ, 722, 1539

Longcope, D. W., Des Jardins, A. C., Carranza-Fulmer, T., \& Qiu, J. 2010, Sol. Phys., 267, 107

Mandrini, C. H., Démoulin, P., Hénoux, J. C., \& Machado, M. E. 1991, A\&A, 250, 541

Oreshina, A. V., \& Somov, B. V. 1998, A\&A, 331, 1078

Oreshina, I. V., \& Somov, B. V. 2006, Astron. Lett., 32, 787

Oreshina, I. V., \& Somov, B. V. 2008, Astron. Notes, 329, 786

Oreshina, I. V., \& Somov, B. V. 2009, Astron. Lett., 35, 207

Parnell, C. E., Haynes, A. L., \& Galsgaard, K. 2010, J. Geophys. Res., 115, A02102

Restante, A. L., Aulanier, G., \& Parnell, C. E. 2009, A\&A, 508, 433

Somov, B. V. 2006, Plasma Astrophysics, Part II, Reconnection and Flares (New York: Springer Science + Business Media, LLC)

Somov, B. V. 2008a, Asian J. Phys., 17, 445

Somov, B. V. 2008b, Astron. Lett., 34, 635

Somov, B. V., \& Titov, V. S. 1985a, Sov. Astron. - AJ, 29, 559

Somov, B. V., \& Titov, V. S. 1985b, Sol. Phys., 102, 79

Somov, B. V., Kosugi, T., Oreshina, I. V., \& Lyubimov, G. P. 2005, Adv. Space Res., 35, 1712

Srivastava, N., Mathew, S. K., \& Louis, R. E. 2009, J. Geophys. Res. 114, CiteID A03107

Sweet, P. 1969, ARA\&A, 7, 149

Syrovatskii, S. I. 1981, ARA\&A, 19, 163

Syrovatskii, S. I., \& Somov, B. V. 1980, Physical driving forces and models of coronal responses, in Solar and Interplanetary Dynamics, ed. M. Dryer, \& E. Tandberg-Hanssen (Dordrecht: Reidel), IAU Symp., 91, 425 